\title{
CDKN2A copy number and p16 expression in malignant pleural mesothelioma in relation to asbestos exposure
}

\author{
Eeva Kettunen ${ }^{1 *} \mathbb{D}$, Sauli Savukoski ${ }^{1}$, Kaisa Salmenkivi², Tom Böhling², Esa Vanhala', Eeva Kuosma',
} Sisko Anttila ${ }^{1,2+}$ and Henrik Wolff ${ }^{1+}$

\begin{abstract}
Background: Deletion of the CDKN2A locus is centrally involved in the development of several malignancies. In malignant pleural mesothelioma (MPM), it is one of the most frequently reported genomic alteration. MPM is strongly associated with a patients' asbestos exposure. However, the status of CDKN2A and the expression of the corresponding protein, p16, in relation to MPM patient's asbestos exposure is poorly known. Copy number alterations in 2p16, 9q33.1 and 19p13 have earlier been shown to accumulate in lung cancer in relation to asbestos exposure but their status in MPM is unclear.
\end{abstract}

Methods: We studied DNA copy numbers for CDKN2A using fluorescence in situ hybridization (FISH) and p16 expression by immunohistochemistry (IHC) in 92 MPM patients, 75 of which with known asbestos exposure status. We also studied, in MPM, copy number alterations in 2p16, 9q33.1 and 19p13 by FISH.

Results: We were unable to detect an association between p16 expression and pulmonary asbestos fiber count in MPM tumor cells. However, significantly more MPM patients with high pulmonary asbestos fiber count (> 1 million fibers per gram [f/g]) had stromal p16 immunoreactivity than MPM of patients with low exposure $(\leq 0.5$ million f/g) $(51.4 \%$ vs 16 . 7\%; $p=0.035$, Chi-Square). We found that an abnormal copy number of CDKN2A in MPM tumor cells associated with a high pulmonary asbestos fiber count ( $p=0.044$, Fisher's Exact test, two-tailed). In contrast to our earlier findings in asbestos associated lung cancer, DNA copy number changes in 2p16, 9q33 and 19p13 were not frequent in MPM although single cases with variable copy numbers on those regions were seen.

Conclusions: We found two instances where the gene locus CDKN2A or its corresponding protein expression, is associated with high asbestos exposure levels. This suggests that there may be biological differences between the mesotheliomas with high pulmonary asbestos fiber count and those with low fiber count.

Keywords: Malignant pleural mesothelioma, CDKN2A, p16, Tumor stroma, Asbestos exposure

\section{Background}

Asbestos fibers have been linked to mechanical and oxidative DNA damage through production of reactive oxidant species and fiber genotoxicity that can arise e.g. as genomic alterations [1-3]. Asbestos have been shown to have an important role in the etiology of some tumors such as malignant mesothelioma, lung cancer and laryngeal

\footnotetext{
* Correspondence: eeva.kettunen@ttl.fi

${ }^{\dagger}$ Anttila Sisko and Wolff Henrik contributed equally to this work.

${ }^{1}$ Research and Service Centre for Occupational Safety, Finnish Institute of Occupational Health, PO Box 40, Fl-00032 Työterveyslaitos, Helsinki, Finland Full list of author information is available at the end of the article
}

cancer $[4,5]$. Alterations of $C D K N 2 A$ locus and its corresponding protein expression are involved in numerous malignancies. In non-small cell lung cancer linked with asbestos exposure $C D K N 2 A$ has been shown to be inactivated, mainly via deletions [6]. CDKN2A/ARF locus encodes tumor suppressor genes $p 16^{I N K 4 A}$ and $p 14^{A R F}$ that interact with cyclin dependent kinase 4 (CDK4) and MDM2 proto-oncogene, respectively, and connect two important oncogenic pathways, $\mathrm{RB}$ and $\mathrm{p} 53$.

Malignant pleural mesothelioma (MPM) is a rare but deadly tumor type that is strongly associated with patients' asbestos exposure [2]. Up to $80-90 \%$ of MPM in men is

C The Author(s). 2019 Open Access This article is distributed under the terms of the Creative Commons Attribution 4.0 International License (http://creativecommons.org/licenses/by/4.0/), which permits unrestricted use, distribution, and 
estimated to be associated with asbestos exposure [7]. In MPM, deletion of $C D K N 2 A$ is the most frequently detected chromosomal change and the most common cause for $\mathrm{p} 16$ protein inactivation (reviewed in [8]). Hypermethylation of $C D K N 2 A$ as a cause of loss of p16 expression in MPM has been reported in a minority of cases $[9,10]$. The frequency of $C D K N 2 A$ deletion in MPM have most often been shown to range from 61 to $88 \%$ in primary tumors, few studies, however, showing deletion only in one-fifth of cases $[9,11-$ 20]. The $C D K N 2 A$ deletions, detected by fluorescence in situ hybridization (FISH), have been exploited in differential diagnosis of MPM and benign mesothelial proliferations on effusions or biopsy material as well as in prognostication aims [13, 16, 20-24]. Expression of p16, however, cannot be used for these purposes [21]. Other genomic alterations (or their protein products) common in MPM such as in BAP1 (BRCA1 associated protein 1), MTAP (methylthioadenosine phosphorylase) and NF2 (neurofibromin 2) have also been studied to find out the most valuable marker combinations for differential diagnosis in MPM [25].

Only few studies - with a relatively limited number of patients - have evaluated the $C D K N 2 A / \mathrm{p} 16$ status in relation to asbestos exposure $[9,11,13,15]$. These studies have shown either no significant association or a rather complex picture, partially the result was dependent on whether the exposure was assessed using occupational history or by asbestos fiber count. In this work we studied the relation between the patients' pulmonary asbestos fiber counts determined by electron microscopy and the CDKN2A/p16 status in 92 MPM. Moreover, we report here for the first time p16 immunoreactivity in MPM in stromal cells and show a significant association between p16 positive stromal staining and asbestos exposure.

In MPM, the asbestos-related genomic changes reported mainly consist of copy number alterations such as deletions in 14q11.2-q21, 6q, 17p, and 22q and DNA methylation changes [26-28]. As we have earlier shown in lung cancer asbestos exposure-related accumulation of copy number alterations in 2p16, 9q33.1 and 19p13 [29, 30], we also studied the same loci in MPM. Contrary to lung cancer, MPM had normal mean copy number in 2p16, 9q33.1 and 19p13. Nevertheless, in these loci the individuals in high asbestos exposure group showed wider ranges of DNA copy number than the low exposure group.

\section{Methods}

\section{Malignant pleural mesothelioma tissue samples}

Study material consisted of 92 formalin-fixed paraffin-embedded (FFPE) MPM specimens from Caucasian patients. Demographic data of the study subjects are shown in Table 1. Pathology samples used for tissue microarrays (for FISH or IHC) have originally been collected in Central Hospitals in Finland as diagnostic specimens. Autopsy specimens for pulmonary fiber count measurements have originally been collected for forensic purposes. Guidelines for this sampling of autopsy specimens require that they represent an area without tumor or fibrosis, at an intermediate distance from bronchus and pleura. Before electron microscopy analysis the samples were also visually inspected at the pathology laboratory of the Finnish Institute of Occupational Health. MPMs displayed either epithelioid, sarcomatoid or biphasic histology. Diagnoses of patients were confirmed by expert pathologists and in borderline cases by consensus [31, 32].

Pulmonary asbestos fiber counts, using fiber length of > $1 \mu \mathrm{m}$, for 75 of the MPM patients could be analyzed using electron microscopy with energy dispersive spectrometry [33] (Table 1). In an international recommendation concerning the attribution of asbestos-related diseases to asbestos exposure, a pulmonary fiber count $>1$ million fibers per gram dry weight of lung tissue $(\mathrm{f} / \mathrm{g})$ is recommended to identify individuals with a high probability of asbestos exposure at work [34]. In this article a pulmonary fiber count $>1$ million $\mathrm{f} / \mathrm{g}$ is referred to as a high exposure and $\geq 5$ million $\mathrm{f} / \mathrm{g}$ to as very high exposure. A pulmonary fiber count $\leq 0.5$ million $\mathrm{f} / \mathrm{g}$ is referred to as a low asbestos exposure. To heighten the contrast between high and low exposure, eight specimens from MPM patients having pulmonary fiber count between 0.5 and 1 million $\mathrm{f} / \mathrm{g}$ were excluded (data not shown in Table 1).

\section{Tissue microarrays}

Tissue microarray (TMA) blocks of MPM specimens were built using Beecher instrument. Four cores of $0.1 \mathrm{~cm}$ in diameter were obtained for each case. If the original sample available was very small, four cores of $0.06 \mathrm{~cm}$ in diameter or minimum two cores of $0.1 \mathrm{~cm}$ in diameter were obtained. TMA blocks included as methodologic quality control specimens some non-tumorous pleura/lung and seven tumors other than malignant mesothelioma (MM) (serous ovarian or serous peritoneal carcinoma from five females, lung adenocarcinoma and pleomorphic liposarcoma from two males). Twenty-six serous ovarian carcinomas in TMA from an earlier study were used as positive p16 expression controls [35].

Routine H\&E staining of sections were studied to evaluate the tumors. Because some sample cores were absent in TMA slides, somewhat different number of tumors produced results in FISH and IHC study, the numbers of tumors being shown in each table.

\section{Immunohistochemistry (IHC)}

For IHC, TMA sections of $2.5 \mu \mathrm{m}$ were treated for antigen retrieval in Lab Vision ${ }^{\text {tm }}$ PT-module (Thermo Fisher Scientific Inc., Fremont, USA) using citrate buffer, pH 6.0 for $10 \mathrm{~min}$ (Thermo Fisher Scientific Inc.). Immunostaining was done in Lab Vision ${ }^{\mathrm{TM}}$ Autostainer (Thermo Fisher Scientific Inc.) using a 1:200 dilution of mouse 
Table 1 Demographic data of the study subjects in MPM microarrays

\begin{tabular}{|c|c|c|c|c|}
\hline & \multicolumn{3}{|c|}{ Malignant pleural mesothelioma $n=92$} & \multirow[t]{3}{*}{ Quality controls ${ }^{b}$} \\
\hline & \multirow{2}{*}{$\begin{array}{l}\text { Asbestos fiber count }^{a} \\
\geq 1.0 \times 10^{6} \mathrm{f} / \mathrm{g} \\
n=53\end{array}$} & \multirow{2}{*}{$\begin{array}{l}\text { Asbestos fiber } \\
\text { count }^{\mathrm{a}} 0-0.5 \times 10^{6} \mathrm{f} / \mathrm{g} \\
n=22\end{array}$} & \multirow{2}{*}{$\begin{array}{l}\text { Fiber count } \\
\text { not available } \\
n=17\end{array}$} & \\
\hline & & & & \\
\hline Gender, male & $50(94 \%)$ & $16(73 \%)$ & $8(47 \%)$ & $2(29 \%)$ \\
\hline Age, mean $\pm S D, y$ & $63.7 \pm 8.7$ & $68.1 \pm 10.2$ & $61.5 \pm 8.3$ & $56.9 \pm 9.4$ \\
\hline Pulmonary fiber count ${ }^{\mathrm{a}}$, median (range), million f/g & $8.9(1.1-1000)$ & $0.2(0-0.5)$ & NA & $0.8(0.4-16.0)^{c}$ \\
\hline \multicolumn{5}{|l|}{ Mesothelioma histologic type } \\
\hline Epithelioid & $43(81 \%)$ & $11(50 \%)$ & $9(53 \%)$ & - \\
\hline Biphasic & $4(8 \%)$ & $6(27 \%)$ & $3(18 \%)$ & - \\
\hline Sarcomatoid & $6(11 \%)$ & $5(23 \%)$ & $5(29 \%)$ & - \\
\hline
\end{tabular}

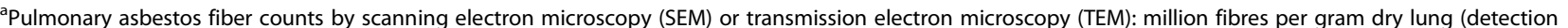
limit $\sim<0.1$ million $\mathrm{f} / \mathrm{g}$ ); ${ }^{\mathrm{b}}$ Methodologic quality controls had either serous ovarian carcinoma, serous ovarian or peritoneal carcinoma, adenocarcinoma of the lung or pleomorphic liposarcoma; ${ }^{C}$ Fiber counts were known for three cases only, thus controls were not used in asbestos-related analysis

monoclonal antibody for p16 (JC8) (Santa Cruz Biotechnology, Inc., Santa Cruz, California, USA) for $60 \mathrm{~min}$ at RT. For detection we used BrightVision plus Poly-HRP-Anti Ms./Rb/Rt IgG (ImmunoLogic, Duiven, Netherlands) with chromogen AEC (Thermo Fisher Scientific Inc.) and Mayer haematoxylin as a background stain. Our p16 staining was further validated by comparing it to that of an accredited medical pathology laboratory (HUSLAB, Helsinki, Finland) p16 staining using $\mathrm{CINtec}^{\circ}$ p16 Histology clone E6H4 ${ }^{\text {pu }}$ (mtm laboratories AG, Roche Diagnostics GmbH, Mannheim, Germany) and DAP. These stainings were made from whole tissue sections of three cases with distinctive staining patterns. The positive controls of twenty-six serous ovarian carcinomas were stained for p16 using CINtec ${ }^{\circ}$ p16 Histology clone $\mathrm{E} 6 \mathrm{H} 4^{\mathrm{rm}}$. Immunostained preparations were reviewed by HW without prior knowledge of the exposure status using an Olympus BH2 (Olympus Europa SE \& Co. KG, Hamburg), and photographed using Leica microscope \& camera system with Leica Application Suite V4.10 [Leica Microsystems (Switzerland) Limited]. Scoring for positivity was as follows: $0-1 \%=$ negative, $2-10 \%=+, 11-50 \%=++$, and $51-100 \%=+++$. For some statistical analysis, the result was dichotomized into negative and positive $(+/++/+++)$ immunoreactivity. Minimum of two TMA cores per sample were required and the highest results among the cores were recorded. Tumor cells and stromal cells were evaluated separately. For sarcomatoid MM, stromal cells were not evaluated. Serous ovarian or serous peritoneal carcinoma from five females, lung adenocarcinoma and pleomorphic liposarcoma from two males served as quality controls in MPM TMAs. Twenty-six serous ovarian carcinomas in a separate TMA served as positive control cases for p16 expression.

\section{Fluorescence in situ hybridization (FISH)}

We studied DNA copy numbers in TMAs for CDKN2A at 9p21 and centromere 9 (CEP9) simultaneously in each cell, using a dual color probe mix of centromeric probe labeled with Spectrum (Sp.) Green and CDKN2A locus specific probe with Sp. Orange (Vysis Inc./ Abbott Molecular Inc., Downers Grove, IL, USA). CDKN2A copy number was considered as abnormal when $>20 \%$ of the cells had lost both CDKN2A signals but showed at least one CEP9 (i.e. homozygous deletion, HD), or $>20 \%$ of the cells had only one CDKN2A signal or at least lower signal number than that of CEP9 (hemizygous deletion), or $\geq 50 \%$ of the cells had one CDKN2A and one CEP9 signal (monosomy).

Locus specific DNA copy numbers in 2p16, 9q33.1 and 19p13 were studied in TMAs using bacterial artificial chromosomes (BAC) as described earlier [36]. Copy numbers of chromosomes 2, 9, 10 and 15 centromeres were studied using a FISHBright CEP9 probe (Qbiogene, Illkirch, France, and Kreatech Biotechnology BV, Amsterdam, The Netherlands), CEP2 alpha satellite DNA probe, Sp. Green-labeled centromere 9 and centromere 10 probes, and Sp. Orange-labeled centromere 15 probe (Vysis Inc./Abbott Molecular, Inc.). Hybridizations were performed according to the manufacturers' instructions. 2p16, 9q33.1, and 19p13 were compared with centromere copy numbers as described earlier [29]. Centromeric probe for chromosome 19 is unavailable, thus 19 p13 signals were divided by an average of centromeric signals of \#2, \#9, \#10, and \#15.

FISH preparations were analysed using a Zeiss AxioImager.Z1 fluorescence microscope (Zeiss, Jena, Germany), without prior knowledge of the exposure status or clinico-pathological data. Efficiency of hybridization was evaluated with lymphocytes serving as internal controls. FISH signals were scored in 30 to 200 non-overlapping interphase nuclei.

\section{Statistical methods}

Chi-Square test and Fisher's Exact test were used to test the differences between asbestos fiber exposure 
groups (very high, high, low). To measure the degree of agreement with $C D K N 2 A$ abnormality and p16 staining we calculated Cohen's Kappa (к) Coefficient.

\section{Results}

MPM tumors from 92 patients having either low $\left(\leq 0.5 \times 10^{6} \mathrm{f} / \mathrm{g}\right)$, high $\left(\geq 1.0 \times 10^{6} \mathrm{f} / \mathrm{g}\right)$, including very high $\left(\geq 5.0 \times 10^{6} \mathrm{f} / \mathrm{g}\right)$, or unknown pulmonary asbestos fiber counts (unknown exposure) were studied for p16 immunoreactivity and CDKN2A copy number. Furthermore, DNA copy numbers were evaluated in genomic regions $2 \mathrm{p} 16,9 \mathrm{q} 33.1$, and $19 \mathrm{p} 13$ that earlier in lung cancer have been found with accumulating changes that associated with asbestos exposure [29]. Some sample cores being absent in some slides, somewhat different number of tumors produced results in FISH and IHC study. The numbers of tumors have been shown in each table. Result for p16 was available for 69 MPM and for both p16 and CDKN2A copy number were available for 62 MPM. For 65 MPM, DNA copy numbers was obtained in one to three of the 2p16, 9q33.1, and 19p13 loci, as proportional to centromere.

\section{Protein expression of p16 in MPM}

Protein expression of p16 was evaluated by IHC in tumor cells and stromal cells in TMA core preparations (Table 2). Three of the MPMs were also studied as an entire tissue section preparation and showed similar staining pattern as in TMA, supporting our approach (Fig. 1). p16 staining status did not associate with gender, age, or mesothelioma histology, neither in MPM tumor nor stromal cells as shown in Table 2. By contrast, significantly more MPM patients with high asbestos exposure had p16 positive stromal staining ( $p=0.035$, Chi-Square) than had MPM of patients with low exposure (51.4\% vs $16.7 \%)$. Positivity of p16 in stromal cells was even more predominant among the very highly exposed when compared with MPM with low exposure $(p=0.018$, Chi-Square $)$ (Table 2). In MPM tumor cells, an association between p16 expression and the asbestos fiber count was not shown.

We also studied the relation of p16 staining between stromal and tumor cells in each individual sample (Table 3). Fifteen (39.5\%) patients with high asbestos count had some staining in MPM stroma but not in tumor cells whereas only one $(8.3 \%)$ patient in low exposure group stained this way $(p=0.048$, Fisher exact test, two-tailed) (Table 3). One of the positive controls stained in this way.
DNA copy number of CDKN2A in relation to centromere 9 (CEP9) in MPM

Using FISH, copy numbers for CDKN2A in 9p21 and CEP9 could be scored in 63 MPM. Homozygous deletion (HD) was the most common abnormality shown in $65 \%$ of the cases. Hemizygosity or monosomy of the chromosome 9 were both shown in $9.5 \%$ whereas $16 \%$ of the MPM had normal copy number. Mean signal counts of CDKN2A and CEP9 were 0.6 and 1.54 in MPM patients with high asbestos exposure whereas in low exposure patients CDKN2A and CEP9 counts were 0.8 and 1.7 (Additional file 1). The proportions of copy number statuses in relation to various clinico-pathological features are shown in Table 2. Abnormal DNA copy number of CDKN2A in MPM tumor cells associated with patients' high pulmonary asbestos fiber count ( $p=0.044$, Fisher's Exact test, two-tailed) (Table 2, Fig. 2). This association was strongest among epithelioid MPM ( $p=$ 0.014, Fisher's Exact test, two-tailed) (Table 2). In Additional files 2 and 4, examples of FISH preparations in MPM with different amount of pulmonary asbestos fibers are shown. The $C D K N 2 A$ copy number did not relate with age of the patient or MPM histologic type. In contrast, there was an association with gender $(p=$ 0.025, Fisher's Exact test, two-tailed) (Table 2) which reflected the higher proportion of subjects with high asbestos exposure among males (76\%) in comparison to females (33\%) (Table 1).

When the relation of CDKN2A abnormality and p16 staining status was observed in individual MPM tumors, low exposure group tumors with abnormal CDKN2A always were negative for p16 staining and vice versa, yet the case numbers were small (Kappa coefficient 1.000) (Additional file 3). Other study groups did not show similar pattern of relation between CDKN2A copy number and p16 staining. Additional file $4(g, h, j)$ shows a sarcomatoid MPM (exposure unknown) with CDKN2A homozygosity in $74 \%$ of the tumor cells and normal copy number of CDKN2A and CEP9 in adjacent hepatic cells, both being negative for p16.

\section{DNA copy numbers in $2 p 16,9 q 33.1,19 p 13$, and chromosome 2, 9, 10, and 15 centromeres}

For 65 MPM, FISH result was obtained in one to three of the 2p16, 9q33.1, and 19p13 loci, mean count of each chromosome centromere serving as a baseline. Mean signal counts of the loci and centromeres \#2, \#9, \#10 and \#15 suggested that copy numbers in 2p16, 9q33.1, and 19p13 and centromeres of chromosomes 2, 9, 10, and 15 in majority of MPM were diploid or close to diploid (Additional file 1). Nevertheless, there were some difference in ranges of the signal counts between the high exposure and low exposure groups as well as in the ranges of mean signal ratios (Additional file 1), the high 


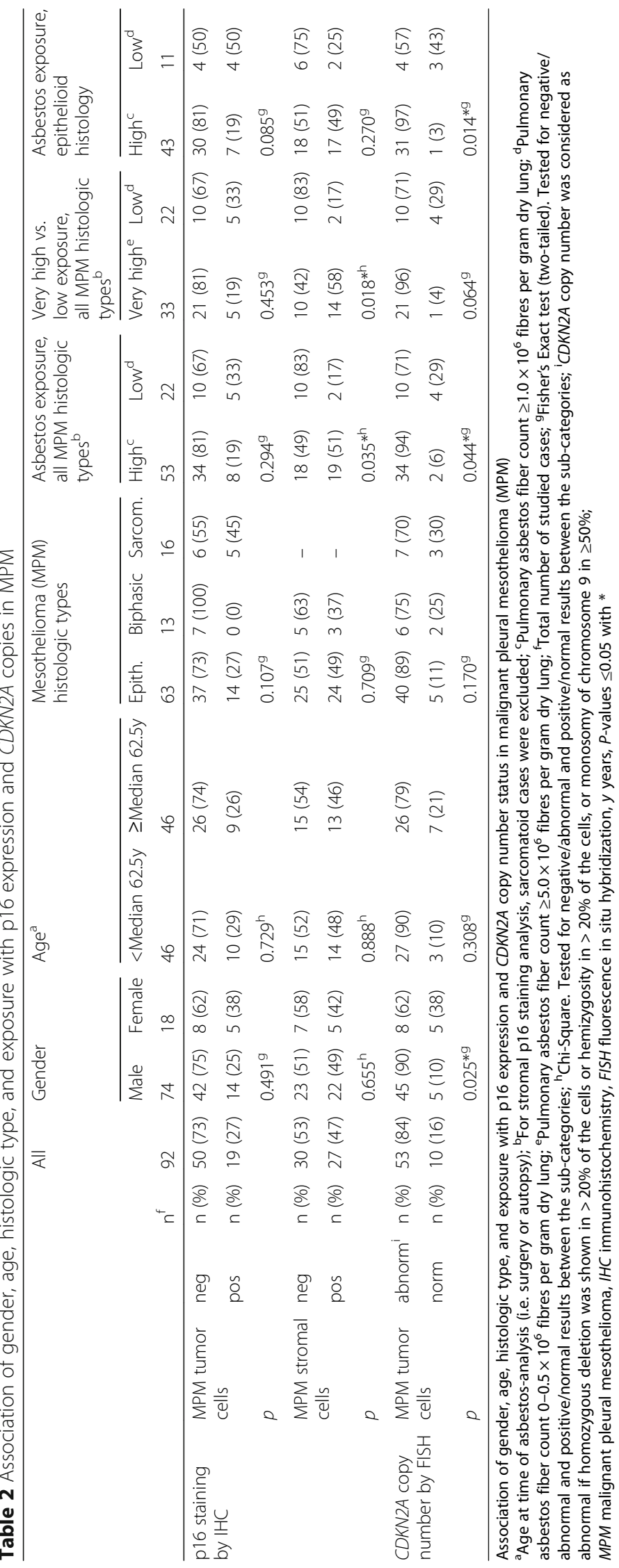




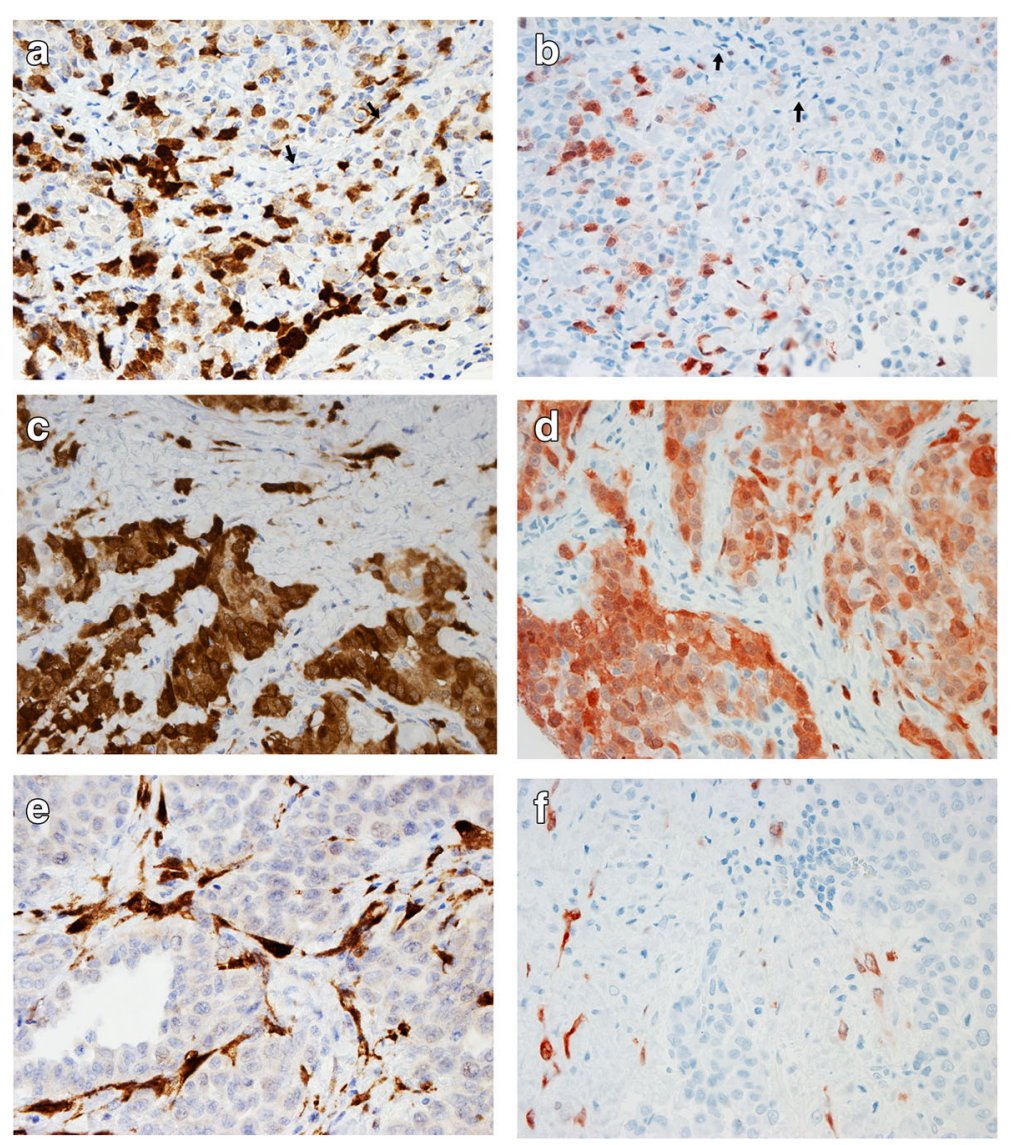

Fig. 1 p16 staining in epithelioid malignant pleural mesothelioma (MPM-E). Both the whole section (a, c, and e; using CINtec ${ }^{\circledR}$ p16 Histology clone E6H4 $4^{T M}$ with DAP as chromogen) and tissue array (TMA) cylinder samples (b, d, and $\mathbf{f}$; using mouse monoclonal antibody for p16 [JC8] with AEC as chromogen) are shown. All microscopic illustrations have magnification $\times 40$. Panels a) and $\mathbf{b}$ ) show p16 staining in the case E-161 who had pulmonary asbestos fiber count of $0.4 \times 10^{6}$ fibers / gram dry weight (f/g). Tumor cells showed positivity of $+++(\mathbf{a})$ to $++(\mathbf{b})$ while stromal cells (arrows) were negative for p16. Panels c) and d) show p16 staining in the case E-143 who had pulmonary asbestos fiber count of $8.9 \times 10^{6} \mathrm{f} / \mathrm{g}$. Tumor cells showed strong positivity and stromal cells had positivity of $++(\mathbf{c})$ to $+(\mathbf{d})$. Panels e) and f) show p16 staining in the case E-250 who had pulmonary asbestos fiber count of $13.0 \times 10^{6} \mathrm{f} / \mathrm{g}$. Tumor cells were negative for p16 whereas stromal cells showed positivity of $+++(\mathbf{e})$ to $++(\mathbf{f})$

exposure group having wider ranges of chromosomal abnormality.

\section{Discussion}

Asbestos exposure causes mechanical and oxidative DNA damage and is linked to genomic alterations $[1,2]$. In mouse studies, MM formation through asbestos exposure has shown a significant role of alterations in Cdkn2a/Arf [37] and deletion of CDKN2A is the most frequently detected chromosomal change in human MPM [8]. Fiber-induced CDKN2A disruption has also been observed in a study in which mesothelioma was induced in mice by instillation of either asbestos fibers or long-fiber carbon nanotube (CNTs) into the pleural cavities [38].

We studied the expression of p16 encoded by $C D K N 2 A$ in MPM from 92 patients with different asbestos-burdens. In the MPM tumor cells, we did not detect an association between p16 expression and asbestos fiber exposure as determined by asbestos fiber count. However, significantly more MPM patients with high pulmonary asbestos fiber count had stromal p16 immunoreactivity than MPM patients with low exposure. The p16 positive stromal staining was often present concurrently with negative p16 staining in tumor cells. To our knowledge earlier studies have not reported p16 immunoreactivity of stromal cells in MPM.

In gynecological malignancies, p16 overexpression in tumor cells is widely used diagnostic marker because it associates with HPV infection indicating a higher risk for cancer. Recently, increased stromal expression of p16, showing gradual increase with the level of malignancy, has been reported in some tumors such as malignant ovarian carcinoma and endometrial carcinoma [39, 40]. However, the significance of stromal p16 immunoreactivity status is not clear. 
Table 3 MPM and controls analyzed in relation with tumor and stromal mark in p16 IHC staining

\begin{tabular}{|c|c|c|c|c|}
\hline \multirow[b]{2}{*}{ p16 staining by tumor - stroma relation } & \multicolumn{2}{|c|}{ Malignant pleural mesothelioma } & \multirow{2}{*}{$\begin{array}{l}\text { Positive controls } \\
n=26^{f}\end{array}$} & \multirow{2}{*}{$\begin{array}{l}\text { Quality controls } \\
n=7\end{array}$} \\
\hline & High exposure $n=47^{\mathrm{b}, \mathrm{c}}$ & Low exposure ${ }^{d} n=17^{e}$ & & \\
\hline \multicolumn{5}{|l|}{ Tumor neg, Stroma neg } \\
\hline n (\%) & $17(45)$ & $7(59)$ & $1(4 \%)$ & $2(\sim 33 \%)^{h}$ \\
\hline$p^{9}$ & 0.774 & & & \\
\hline \multicolumn{5}{|l|}{ Tumor pos, Stroma neg } \\
\hline n (\%) & $2(5)$ & $3(25)$ & $4(15 \%)$ & $2(\sim 33 \%)^{\mathrm{i}}$ \\
\hline$p^{9}$ & 0.112 & & & \\
\hline \multicolumn{5}{|l|}{ Tumor neg, Stroma pos } \\
\hline n (\%) & $15(40)$ & $1(8)$ & $1(4 \%)$ & $0(0 \%)$ \\
\hline$p^{9}$ & $0.048^{*}$ & & & \\
\hline \multicolumn{5}{|l|}{ Tumor pos, Stroma pos } \\
\hline n (\%) & $4(10)$ & $1(8)$ & $20(77 \%)$ & $2(\sim 33 \%)^{j}$ \\
\hline$p^{9}$ & 1.000 & & & \\
\hline
\end{tabular}

Epithelioid and biphasic malignant pleural mesothelioma and controls analyzed in relation with tumor and stromal mark in p16 immunohistochemical (IHC) staining. ${ }^{a}$ Pulmonary asbestos fiber count $\geq 1.0 \times 10^{6}$ fibres per gram dry lung $(\mathrm{f} / \mathrm{g})$; ${ }^{\mathrm{b}}$ For two cases, tumor stained positive but stroma could not be analyzed; ${ }^{\mathrm{C}}$ For eight cases IHC staining was not available; ${ }^{d}$ Pulmonary asbestos fiber count $0-0.5 \times 10^{6} \mathrm{f} / \mathrm{g}$; ${ }^{\mathrm{C}}$ For five cases IHC staining was not available; ${ }^{f}$ Positive controls were serous ovarian carcinomas; ${ }^{9}$ Fisher's Exact Test, two-tailed; ${ }^{\text {h }}$ Adenocarcinoma of the lung and pleomorphic liposarcoma; 'Serous ovarian carcinoma and serous

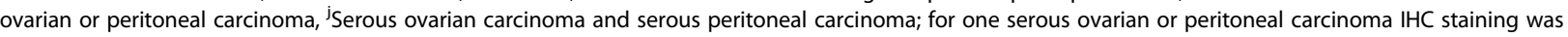
not available.

It has been shown that increased expression of p16 could induce senescence without a senescence-associated secretory phenotype and limit the accumulation of DNA damage [41]. Our result of stromal p16 expression in highly asbestos exposed patients' MPM may mark a response to cell activation caused by fibers but further studies are needed. Mouse studies have produced experimental data about some molecular changes related to fiber exposure as described above [37, 38] whereas in human MPM asbestos-related changes reported are mainly copy number alterations and DNA methylation changes [26-28]. There are some reports suggesting that mesotheliomas associated with asbestos exposure have a poorer prognosis than those not associated with the exposure [42-45].

The most frequently reported chromosomal alteration in MPM $(61 \%$ - 88\%) is deletion of material in 9p21, i.e. primarily $C D K N 2 A$ encoding tumor suppressors p16INK4A and p14ARF $[9,11-13,15,16,18-20]$. Accordingly, a majority $(84.1 \%)$ of our MPM cases showed a $C D K N 2 A$ copy number abnormality, either HD or hemizygous deletion or monosomy. The LSI CDKN2A probe we utilized covers also CDKN2B and MTAP encoding sequences in 9p21. In line with our result, a comprehensive cytogenetic study of $17 \mathrm{MM}$ cell lines has shown that the above-mentioned genes in the heart of the region showed HD in $82-100 \%$ of the cell lines [46]. Moreover, there were numerous other genes in $9 \mathrm{p} 21$ showing HD or allelic loss in $12-65 \%$ of those MM cell lines and other additional complex chromosome 9 rearrangements being identified by combined chromosomal techniques [46].
In our study, asbestos exposure of the MPM patients has been evaluated using pulmonary asbestos fiber counts measured with electron microscopy. We showed that abnormal copy number of CDKN2A in MPM tumor cells associated with high pulmonary asbestos fiber count but not with age or MPM histologic type. An earlier study of Hirao et al. showed a somewhat complex relation between asbestos exposure and $p 16$ changes. In their study, $p 16$ alterations (deletion or methylation) were more common in cases with lower pulmonary asbestos (fiber counts by a method different than ours) although $p 16$ deletions were more common in heavy exposed group. In that study, part of asbestos exposure of the subjects had been evaluated by medical or occupational history [15]. In other earlier studies, exposure data obtained from work history evaluation, $C D K N 2 A$ deletion and asbestos exposure did not associate with each other $[9,11,13]$.

We found here centromere 9 (CEP9) to be diploid when using the single probe. With the dual probe mix including probes for CEP9 and CDKN2A, somewhat lowered mean CEP9 signals were counted. This could be due to the different probe used in these two analysis types and heterogeneous specimens of genetically disturbed cancer cells with variable changes. When establishing cut-off values for CDKN2A FISH protocol, Chung et al. have shown a pattern of 'one CDKN2A signal/one CEP9 signal' in $6-34 \%$ of nuclei in reactive mesothelial proliferation and further in $5 \%$ of nuclei in MPM [13]. In MM cell lines, chromosome 9 fragments rather than whole 9 have been shown affected by HD, chromosomal aberrations being though various and very 


\section{a}

DNA copy number vs. pulmonary fiber count

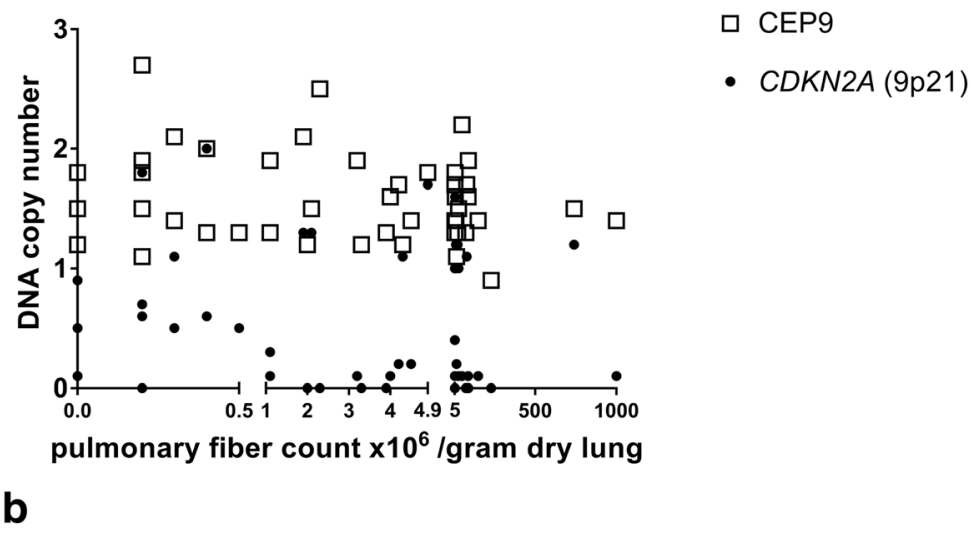

CDKN2A/CEP9 vs. pulmonary fiber count

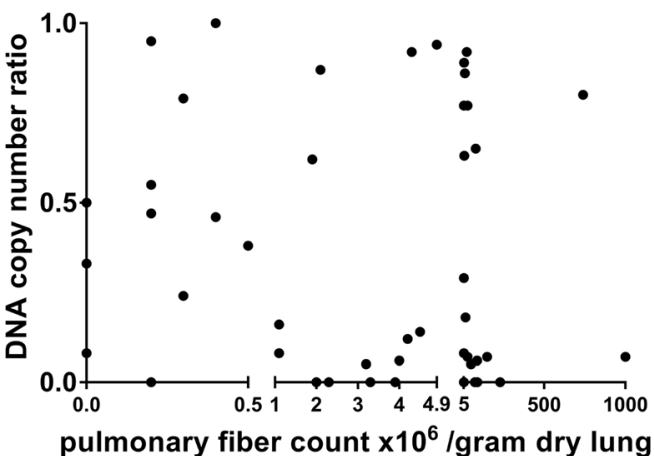

Fig. 2 Scatter plots showing the relation of the pulmonary asbestos fiber count in each individual suffering from malignant pleural mesothelioma and $\mathbf{a}$ ) the mean DNA copy numbers of CDKN2A at 9p21 and the centromeric locus of chromosome 9 (CEN9) or b) the ratio of the mean DNA copy numbers of CDKN2A and CEN9 in the tumors of the patients. The $x$-axis scale is discontinuous to provide dynamic range from the low $\left(\leq 0.5 \times 10^{6} \mathrm{f} / \mathrm{g}\right)$ to high $\left(\geq 1.0 \times 10^{6} \mathrm{f} / \mathrm{g}\right)$ and to very high fiber counts $\left(\geq 5.0 \times 10^{6} \mathrm{f} / \mathrm{g}\right)$

complex in MM [46]. Our CEP9 results are in line with those reports.

This is the first report indicating a correlation between the $C D K N 2 A$ deletion status and the p16 protein levels in MPM patients having low asbestos exposure. Earlier, somewhat discrepant results about the relationship and correlation of the CDKN2A deletion status and the p16 levels in MPM has been reported [9, 14, 20, 21]. Immunoreactivity for the proteins p14, p15, p16 and MTAP, all encoded from DNA in 9p21, has been studied and compared with FISH result of $9 \mathrm{p} 21$. MTAP protein expression has been reported to correlate best with HD of $C D K N 2 A$ [47]. There are also other mechanisms such as DNA methylation that can influence protein expression [10]. In our study, the $C D K N 2 A$ probe in 9 p21 we used covers also MTAP and CDKN2B whereas the antibody in immunohistochemistry recognized specifically CDKN2A/p16. This could cause some lack of correlation in the high exposure MPM and controls. Moreover, $C D K N 2 A$ copy number in majority of MPM is abnormal and thus there are relatively small numbers of cases with normal $C D K N 2 A$ copy number available for a statistical comparison. Nevertheless, the correlation between the CDKN2A deletion status and p16 protein levels was shown here for the first time, interestingly only in the low exposure group MPM. The significance of this finding is unclear, but it is in line with the other findings in this paper that suggest differences in various aspects of $C D K N 2 A / p 16$ depending on the level of asbestos exposure.

Our studies here on locus specific DNA copy numbers and on the centromeres of chromosomes 2, 9, 10 and 15 suggested that asbestos exposure related accumulation of alterations in 2p16, 9q33 and 19p13 that we earlier have showed in lung cancer $[29,30]$ was not comparable in MPM. Yet we found out that the ranges of signal count in 19p13 and 9q33 in MPM were wider with high exposure group than with low exposure group despite the normal mean signal count. Abnormal signals, even if being infrequent may suggest that in asbestos-exposed 
patients these genomic regions gained disintegration due to exposure-induced DNA damage, reflected as increased locus copy number variation in single MPM individuals.

Interestingly, loss in 19p13.2 coinciding with the sequence that is recognized by our $19 \mathrm{p} 13$ probe has earlier been reported in 55\% of MPM but without an association to asbestos exposure probably due to the small number of non-exposed patients in that study [48]. Although $2 \mathrm{p}$ changes were not reported using comparative genomic hybridization in primary MPM tumors [49], another study on MPM cell lines have shown gains in 2p16.2-p12, 2p11.2-q11.2, and 19p13.13, depending on the cell passage [50].

\section{Conclusions}

In conclusion, we report here for the first time that p16 immunoreactivity in stromal cells of MPM had significant relation with the patients' high asbestos exposure, suggesting that the biology of MPM in highly exposed individuals differed from that of mesotheliomas in subjects with low exposure. Additionally, abnormal copy number of CDKN2A in MPM tumor cells associated with high pulmonary asbestos fiber count, again suggesting a biological difference between mesotheliomas depending on the degree of asbestos exposure. In contrast to the situation in lung cancer, asbestos exposure associated molecular changes in 2p16, 9q33 and $19 \mathrm{p} 13$ were not frequent in MPM though single cases with variable DNA copy numbers on those regions were seen.

\section{Additional files}

Additional file 1: A table - Mean signal counts of fluorescent locus specific probes and centromeric CEP probes in different study groups of malignant pleural mesothelioma in FISH analysis. (PDF $703 \mathrm{~kb}$ )

Additional file 2: Figure legend for Additional file 4. (PDF $1005 \mathrm{~kb}$ )

Additional file 3: A table - Relation of the CDKN2A copy number and p16 staining status in tumor cells of malignant pleural mesothelioma patients in general and with different asbestos-burden and in quality controls. (PDF $762 \mathrm{~kb}$ )

Additional file 4: A pdf file of images of FISH and IHC preparations of malignant pleural mesothelioma (MPM). (PDF $2607 \mathrm{~kb}$ )

\section{Abbreviations}

BAC: Bacterial artificial chromosome; BAP1: BRCA1 associated protein 1; CDK4: Cyclin dependent kinase 4; CEP: Centromere; CNT: Carbon nanotube; f/g: Fibers per gram; FFPE: Formalin-fixed paraffin-embedded;

FISH: Fluorescence in situ hybridization; HD: Homozygous deletion; IHC: Immunohistochemistry; MM: Malignant mesothelioma; MPM: Malignant pleural mesothelioma; MTAP: Methylthioadenosine phosphorylase; NF2: Neurofibromin 2; Sp: Spectrum; TMA: Tissue microarray

\section{Acknowledgements}

We thank Helinä Hämäläinen and Päivi Tuominen for excellent technical assistance. We are very grateful to Dr. Ralf Bützow for providing the TMA of ovarian serous adenocarcinomas.

\section{Funding}

This work has been financially supported by the Academy of Finland Grant no. 115372 to E. Kettunen, the K. Albin Johansson Foundation to E. Kettunen, and the Finnish Work Environment Fund no. 109003 to Sisko Anttila. The funding sources have not been involved in the design of the study, collection, analysis, and interpretation of data, or in writing the manuscript.

\section{Availability of data and materials}

The datasets used and analysed during the current study are available from the corresponding author on reasonable request.

\section{Authors' contributions}

EKe, SA and HW have designed the study. SA organized the collecting of the specimens. SA, TB, KS, and HW verified the histological diagnoses. EV performed the pulmonary fiber analyses. EKe and SS performed the laboratory work. EKe analyzed the FISH preparations and HW the immunohistochemistry preparations. EKu performed the statistical analyses. EKe and HW have written the first draft of the manuscript to which all the authors then contributed and approved the submitted version.

\section{Ethics approval and consent to participate}

The Coordinating Ethical Review Board, Helsinki and Uusimaa Hospital District and the Ethical Review Board for Research in Occupational Health and Safety, Helsinki, Finland, approved the collection of specimens, the study protocol, and the use of the specimens and asbestos fiber analysis results, obtained originally for diagnostic purposes. The patients are deceased, consent from them was not required (Dnro 39/13/03/00/09; Dnro 105/13/03/ 00/2013, Dnro TTL/2/2016; Dnro THL/1055/5.05.01/2013; Dnro 5929/

06.01.03.01/2013).

\section{Consent for publication}

Not applicable.

\section{Competing interests}

The authors declare that they have no competing interests.

\section{Publisher's Note}

Springer Nature remains neutral with regard to jurisdictional claims in published maps and institutional affiliations.

\section{Author details}

${ }^{1}$ Research and Service Centre for Occupational Safety, Finnish Institute of Occupational Health, PO Box 40, FI-00032 Työterveyslaitos, Helsinki, Finland. ${ }^{2}$ Department of Pathology, University of Helsinki, and HUSLAB, Helsinki University Hospital, 00029 HUS, Helsinki, Finland.

Received: 9 November 2018 Accepted: 29 April 2019

Published online: 28 May 2019

\section{References}

1. Jaurand M. Mechanisms of fiber-induced genotoxicity. Environ Health Perspect. 1997;105(Suppl 5):1073-84.

2. Mossman BT, Shukla A, Heintz NH, Verschraegen CF, Thomas A, Hassan R. New insights into understanding the mechanisms, pathogenesis, and management of malignant mesotheliomas. Am J Pathol. 2013;182:1065-77.

3. Sekido Y. Molecular pathogenesis of malignant mesothelioma. Carcinogenesis. 2013;34:1413-9.

4. $\quad$ LaDou J. The asbestos cancer epidemic. Environ Health Perspect. 2004;112: 285-90.

5. Wolff H, Vehmas T, Oksa P, Rantanen J, Vainio H. Asbestos, asbestosis, and cancer, the Helsinki criteria for diagnosis and attribution 2014: recommendations. Scand J Work Environ Health. 2015;41:5-15.

6. Andujar P, Wang J, Descatha A, Galateau-Sallé F, Abd-Alsamad I, BillonGalland M-A, Blons H, Clin B, Danel C, Housset B, et al. p16INK4A inactivation mechanisms in non-small-cell lung cancer patients occupationally exposed to asbestos. Lung Cancer. 2010;67:23-30.

7. Travis WD, Brambilla E, Burke AP, Marx A, Nicholson AG, editors. WHO classification of tumours of the lung, pleura, thymus and heart. 4th ed. Lyon: IARC; 2015.

8. Kettunen E, Knuutila S. Malignant mesothelioma, molecular markers. In: Anttila SL, Boffetta P, editors. Occupational cancers. London: Springer; 2014. p. 325-43. 
9. Kobayashi N, Toyooka S, Yanai H, Soh J, Fujimoto N, Yamamoto H, Ichihara S, Kimura K, Ichimura K, Sano Y, et al. Frequent p16 inactivation by homozygous deletion or methylation is associated with a poor prognosis in Japanese patients with pleural mesothelioma. Lung Cancer. 2008;62:120-5.

10. Wong L, Zhou J, Anderson D, Kratzke R. Inactivation of p16INK4a expression in malignant mesothelioma by methylation. Lung Cancer. 2002;38:131-6.

11. Bahnassy AA, Zekri AR, Abou-Bakr AA, El-Deftar MM, El-Bastawisy A, Sakr MA, El-Sherif GM, Gaafar RM. Aberrant expression of cell cycle regulatory genes predicts overall and disease free survival in malignant pleural mesothelioma patients. Exp Mol Pathol. 2012;93:154-61.

12. Cheng JQ, Jhanwar SC, Klein WM, Bell DW, Lee W-C, Altomare DA, Nobori T, Olopade OI, Buckler AJ, Testa JR. p16 alterations and deletion mapping of 9p21-p22 in malignant mesothelioma. Cancer Res. 1994;54:5547-51.

13. Chung CT, Santos Gda C, Hwang DM, Ludkovski O, Pintilie M, Squire JA, Tsao MS. FISH assay development for the detection of p16/CDKN2A deletion in malignant pleural mesothelioma. J Clin Pathol. 2010;63:630-4.

14. Dacic S, Kothmaier H, Land S, Shuai Y, Halbwedl I, Morbini P, Murer B, Comin C, Galateau-Salle F, Demirag F, et al. Prognostic significance of p16/cdkn2a loss in pleural malignant mesotheliomas. Virchows Arch. 2008:453:627-35.

15. Hirao T, Bueno R, Chen C, Gordon G, Heilig E, Kelsey K. Alterations of the p16(INK4) locus in human malignant mesothelial tumors. Carcinogenesis. 2002;23:1127-30.

16. Illei PB, Rusch WW, Zakowski MF, Ladanyi M. Homozygous deletion of CDKN2A and codeletion of the methylthioadenosine phosphorylase gene in the majority of pleural mesotheliomas. Clin Cancer Res. 2003;9:2108-13.

17. Lopez-Rios F, Chuai S, Flores R, Shimizu S, Ohno T, Wakahara K, Illei PB, Hussain S, Krug L, Zakowski MF, et al. Global gene expression profiling of pleural mesotheliomas: overexpression of Aurora kinases and P16/CDKN2A deletion as prognostic factors and critical evaluation of microarray-based prognostic prediction. Cancer Res. 2006;66:2970-9.

18. Monaco SE, Shuai Y, Bansal M, Krasinskas AM, Dacic S. The diagnostic utility of p16 FISH and GLUT-1 immunohistochemical analysis in mesothelial proliferations. Am J Clin Pathol. 2011;135:619-27.

19. Papp T, Schipper H, Pemsel H, Bastrop R, Müller K, Wiethege T, Weiss D, Dopp E, Schiffmann D, Rahman Q. Mutational analysis of N-ras, p53, p16INK4a, p14ARF and CDK4 genes in primary human malignant mesotheliomas. Int J Oncol. 2001;18:425-33.

20. Takeda M, Kasai T, Enomoto Y, Takano M, Morita K, Kadota E, Nonomura A. 9 p21 deletion in the diagnosis of malignant mesothelioma, using fluorescence in situ hybridization analysis. Pathol Int. 2010;60:395-9.

21. Chiosea S, Krasinskas A, Cagle PT, Mitchell KA, Zander DS, Dacic S. Diagnostic importance of 9p21 homozygous deletion in malignant mesotheliomas. Mod Pathol. 2008;21:742-7.

22. Flores-Staino C, Darai-Ramqvist E, Dobra K, Hjerpe A. Adaptation of a commercial fluorescent in situ hybridization test to the diagnosis of malignant cells in effusions. Lung Cancer. 2010;68:39-43.

23. Onofre FB, Onofre AS, Pomjanski N, Buckstegge B, Grote HJ, Bocking A. 9 p21 deletion in the diagnosis of malignant mesothelioma in serous effusions additional to immunocytochemistry, DNA-ICM, and AgNOR analysis. Cancer. 2008;114:204-15.

24. Savic S, Franco N, Grilli B, Barascud Ade V, Herzog M, Bode B, Loosli H, Spieler $\mathrm{P}$, Schonegg $\mathrm{R}$, Zlobec I, et al. Fluorescence in situ hybridization in the definitive diagnosis of malignant mesothelioma in effusion cytology. Chest. 2010;138:137-44.

25. Berg KB, Dacic S, Miller C, Cheung S, Churg A. Utility of methylthioadenosine phosphorylase compared with BAP1 immunohistochemistry, and CDKN2A and NF2 fluorescence in situ hybridization in separating reactive mesothelial proliferations from epithelioid malignant mesotheliomas. Arch Pathol Lab Med. 2018;142(12):1549-53.

26. Borczuk AC, Pei J, Taub RN, Levy B, Nahum O, Chen J, Chen K, Testa JR. Genome-wide analysis of abdominal and pleural malignant mesothelioma with DNA arrays reveals both common and distinct regions of copy number alteration. Cancer Biol Ther. 2016;17:328-35.

27. Christensen B, Houseman E, Godleski J, Marsit C, Longacker J, Roelofs C, Karagas M, Wrensch M, Yeh R, Nelson $H$, et al. Epigenetic profiles distinguish pleural mesothelioma from normal pleura and predict lung asbestos burden and clinical outcome. Cancer Res. 2009;69:227-34.

28. Jean D, Thomas E, Manie E, Renier A, de Reynies A, Lecomte C, Andujar P, Fleury-Feith J, Galateau-Salle F, Giovannini M, et al. Syntenic relationships between genomic profiles of fiber-induced murine and human malignant mesothelioma. Am J Pathol. 2011;178:881-94.
29. Nymark P, Aavikko M, Mäkilä J, Ruosaari S, Hienonen-Kempas T, Wikman H, Salmenkivi K, Pirinen R, Karjalainen A, Vanhala $\mathrm{E}_{1}$ et al. Accumulation of genomic alterations in 2p16,9q33.1 and 19p13 in lung tumours of asbestos-exposed patients. Mol Oncol. 2013;7:29-40.

30. Nymark P, Wikman H, Ruosaari S, Hollmén J, Vanhala E, Karjalainen A, Anttila S, Knuutila S. Identification of specific gene copy number changes in asbestos-related lung cancer. Cancer Res. 2006;16:5737-43.

31. Butnor KJ. My approach to the diagnosis of mesothelial lesions. J Clin Pathol. 2006;59:564-74.

32. Travis WD. Sarcomatoid neoplasms of the lung and pleura. Arch Pathol Lab Med. 2010;134:1645-58.

33. Tuomi T. Fibrous minerals in the lungs of mesothelioma patients: comparison between data on SEM, TEM, and personal interview information. Am J Ind Med. 1992;21:155-62.

34. Tossavainen A. A reporter. Asbestos, asbestosis, and cancer: the Helsinki criteria for diagnosis and attribution. Scand J Work Environ Health. 1997;23:311-6.

35. Lassus H, Leminen A, Lundin J, Lehtovirta P, Bützow R. Distinct subtypes of serous ovarian carcinoma identified by p53 determination. Gynecol Oncol. 2003:91:504-12.

36. Kettunen E, Aavikko M, Nymark P, Ruosaari S, Wikman H, Vanhala E, Salmenkivi K, Pirinen R, Karjalainen A, Kuosma E, et al. DNA copy number loss and allelic imbalance at 2p16 in lung cancer associated with asbestos exposure. Br J Cancer. 2009;100:1336-42.

37. Altomare DA, Menges CW, XU J, Pei J, Zhang L, Tadevosyan A, NeumannDomer E, Liu Z, Carbone M, Chudoba I, et al. Losses of both products of the Cdkn2a/Arf locus contribute to asbestos-induced mesothelioma development and cooperate to accelerate tumorigenesis. PLoS One. 2011;6:e18828.

38. Chernova T, Murphy FA, Galavotti S, Sun XM, Powley IR, Grosso S, Schinwald A, Zacarias-Cabeza J, Dudek KM, Dinsdale D, et al. Long-fiber carbon nanotubes replicate asbestos-induced mesothelioma with disruption of the tumor suppressor gene Cdkn2a (Ink4a/Arf). Curr Biol. 2017;27:3302-3314 e3306.

39. Yoon G, Koh CW, Yoon N, Kim JY, Kim HS. Stromal p16 expression is significantly increased in endometrial carcinoma. Oncotarget. 2017;8:4826-36.

40. Yoon N, Yoon G, Park CK, Kim HS. Stromal p16 expression is significantly increased in malignant ovarian neoplasms. Oncotarget. 2016;7:64665-73.

41. Coppe JP, Rodier F, Patil CK, Freund A, Desprez PY, Campisi J. Tumor suppressor and aging biomarker p16(INK4a) induces cellular senescence without the associated inflammatory secretory phenotype. J Biol Chem. 2011;286:36396-403.

42. Christensen BC, Godleski JJ, Roelofs CR, Longacker JL, Bueno R, Sugarbaker DJ, Marsit CJ, Nelson HH, Kelsey KT. Asbestos burden predicts survival in pleural mesothelioma. Environ Health Perspect. 2008;116:723-6.

43. Flores RM, Zakowski M, Venkatraman E, Krug L, Rosenzweig K, Dycoco J, Lee C, Yeoh C, Bains M, Rusch V. Prognostic factors in the treatment of malignant pleural mesothelioma at a large tertiary referral center. J Thorac Oncol. 2007;2:957-65.

44. Leuzzi G, Rea F, Spaggiari L, Marulli G, Sperduti I, Alessandrini G, Casiraghi M, Bovolato P, Pariscenti G, Alloisio M, et al. Prognostic score of long-term survival after surgery for malignant pleural mesothelioma: a multicenter analysis. Ann Thorac Surg. 2015;100:890-7.

45. Madrigal C, Aguilar G, Juárez CA, Trujillo O, Jiménez C, Trejo LA, Fierro D, Calderón ES. 1382 survival analysis of malignant pleural mesothelioma in mexican workers. OEMED. 2018;75:A455.

46. Klorin G, Rozenblum E, Glebov O, Walker RL, Park Y, Meltzer PS, Kirsch IR, Kaye FJ, Roschke AV. Integrated high-resolution array CGH and SKY analysis of homozygous deletions and other genomic alterations present in malignant mesothelioma cell lines. Cancer Genet. 2013;206:191-205.

47. Hida T, Hamasaki M, Matsumoto S, Sato A, Tsujimura T, Kawahara K, Iwasaki A, Okamoto T, Oda Y, Honda H, et al. Immunohistochemical detection of MTAP and BAP1 protein loss for mesothelioma diagnosis: comparison with 9p21 FISH and BAP1 immunohistochemistry. Lung Cancer. 2017;104:98-105.

48. Ivanov SV, Miller J, Lucito R, Tang C, Ivanova AV, Pei J, Carbone M, Cruz C, Beck A, Webb C, et al. Genomic events associated with progression of pleural malignant mesothelioma. Int J Cancer. 2009;124:589-99.

49. Lindholm P, Salmenkivi K, Vauhkonen H, Nicholson A, Anttila S, Kinnula V, Knuutila S. Gene copy number analysis in malignant pleural mesothelioma using oligonucleotide array CGH. Cytogen Genome Res. 2007;119:46-52.

50. Zanazzi C, Hersmus R, Veltman I, Gillis A, van Drunen E, Beverloo H, Hegmans J, Verweij M, Lambrecht B, Oosterhuis J, et al. Gene expression profiling and gene copy-number changes in malignant mesothelioma cell lines. Genes Chrom Cancer. 2007;46:895-908. 